\title{
Conectando los Espacios de Trabajo Aritmético y Geométrico a través de la noción de aproximación en Geogebra Connecting arithmetic and geometric workspaces through the notion of approximation in Geogebra
}

\author{
María de Lourdes Guerrero Magaña \\ Universidad de Guadalajara \\ Lourdes.Guerrero@gmail.com \\ Rafael Pantoja Rangel \\ Universidad de Guadalajara \\ rafael.pantoja@red.cucei.udg.mx
}

\section{Resumen}

A través de la presente investigación se analizó la aproximación de medidas en objetos geométricos con Geogebra en el nivel bachillerato, y los resultados mostraron que los estudiantes de bachillerato tienen débiles nociones del concepto de aproximación no obstante sus vastos recursos aritméticos. Dichos resultados permitieron, por un lado, analizar la potencialidad que tienen para el aprendizaje al conectar ideas geométricas y aritméticas y, por otro lado, entender mejor sus fortalezas y dificultades. Enmarcamos este trabajo en la teoría de representaciones de Duval (1993), así como en los trabajos de Núñez y Cortés (2008) sobre Ambientes Tecnológicos Interactivos para el Aprendizaje de las Matemáticas (ATIAM), y de Kuzniak (2012, 2013), sobre la importancia de transitar en diferentes campos de las matemáticas.

Palabras clave: Conectando conceptos de aritmética y geometría, Geogebra, Medición, Geometría. 


\section{Abstract}

Through this research we analyzed the measures approximation in geometric objects with Geogebra ant high school level, and the results showed that high school students have weak notions of the concept of approximation, however its vast resources of arithmetic. These results permitted, on the one hand, analyze the potential they have for learning by connecting ideas geometric and arithmetic, and, on the other hand, better understand their strengths and difficulties. We frame this work in the theory of Duval representations (1993), as well as in the work of Núñez and Cortés (2008) on Interactive Technological Environments for Math Learning (ATIAM), and Kuzniak $(2012,2013)$, on the importance of transit in different Areas of mathematics.

Keywords: Connecting concepts of arithmetic and geometry, Geogebra, geometry.

Fecha Recepción: $\quad$ Septiembre $2014 \quad$ Fecha Aceptación: Noviembre 2014

\section{Introducción}

La globalización ha hecho que los estudiantes de ahora deban tener ciertas competencias que les permitan enfrentarse a nuevas situaciones; para ello, el joven requiere de una formación integral basada en una actualización constante y dinámica que se adapte a los distintos cambios de la sociedad. De esa manera, el método de enseñanza tradicional, junto con el conductismo de los años ochenta, ha dejado de ser lo que requiere el alumno.

En este mismo contexto, el avance constante en el uso de las Tecnologías de la Información y la Comunicación (TIC) en todos los ámbitos de nuestra vida, ha vuelto prioritario innovar los métodos de enseñanza-aprendizaje.

El uso de la tecnología ha permitido el manejo dinámico-interactivo de múltiples sistemas de representación, lo que ha repercutido en un aprendizaje que pone más énfasis en los conceptos y el entendimiento matemático. Asimismo, las TIC han permitido que los estudiantes comprendan mejor los problemas y dificultades a los que se enfrentan en el aprendizaje de ciertos conceptos matemáticos. 
Las primeras experiencias con el uso de software en los salones de clase de matemáticas (laboratorios) fue una tarea ardua, que muchas veces trajo consigo más desventajas que beneficios, debido, entre otras cosas, al alto costo de las computadoras. Asimismo, el software desarrollado era poco amigable y transparente, con tiempos de ejecución prolongados, lo que producía experiencias didácticas poco útiles desde el punto de vista del aprendizaje matemático. Sin embargo, a lo largo del tiempo se han ido actualizando tanto hardware como software. Este último ha servido como punto de apoyo para el desarrollo de la ciencia y la tecnología. Los especialistas lo han adaptado, haciéndolo más eficiente y especializado para poder utilizarlo en ramas específicas de la enseñanza; por ejemplo, las matemáticas.

Los beneficios vislumbrados sobre el uso de la tecnología en los procesos de enseñanza y aprendizaje de las matemáticas, son considerables. Uno de ellos es la posibilidad de manejar dinámicamente objetos matemáticos en múltiples registros de representación dentro de esquemas interactivos, difíciles de lograr con los medios tradicionales no fácilmente manipulables. Así, el conocimiento matemático obtenido a través de la exploración de los objetos asume características no tradicionales.

Por todo ello, la presente investigación se sustentó en la necesidad de buscar formas en las que los estudiantes de bachillerato, mediante el uso de herramientas tecnológicas y de actividades de aprendizaje, generaran un aprendizaje conceptual acerca de las nociones de aproximación y estimación en ambientes de aprendizaje interactivos. Dichas nociones, si bien se relacionan con el estudio de la aritmética, que los estudiantes estudian prácticamente a lo largo de toda la primaria y parte de la secundaria en nuestro país, son escasamente tratadas en el currículo y en el aula. Esta situación la comparten estudiantes de otros países, por lo que varios investigadores (Chang, Males, Mosier \& Ginulates, 2011; Gooya, Khosroshahi \& Teppo, 2011; Hannighofer, Van den Heuvel-Panhuizen, Weirich \& Robitzsch, 2011; Smith, Van den Heuvel-Panhuizen \& Teppo, 2011) han resaltado la necesidad de utilizar actividades que favorezcan el desarrollo de estas habilidades. 


\section{Objetivo}

El objetivo primario de la investigación fue tener un mejor entendimiento de los procesos que siguen los estudiantes y los recursos matemáticos que utilizan cuando trabajan con Geogebra en actividades de aproximación que buscan combinar de manera natural dos áreas básicas en las matemáticas escolares: la geometría y la aritmética.

Algunos objetivos adicionales fueron:

- Disponer de información bien documentada sobre las fortalezas y dificultades de estudiantes de bachillerato en el aprendizaje de la noción de aproximación.

- Generar actividades de aprendizaje conceptual que apoyen el uso de los recursos tecnológicos en el aula de matemáticas, particularmente de la utilización de software educativo de geometría dinámica.

Para lograr estos objetivos se plantearon las siguientes preguntas de investigación: ¿Qué características del software Geogebra permiten generar un ambiente de aprendizaje en el que los estudiantes logren desarrollar habilidades para estimar y aproximar cantidades numéricas? ¿Qué importancia da el estudiante al uso de software educativo para el aprendizaje de las matemáticas? ¿Y qué estrategias y recursos matemáticos utilizaron los estudiantes para enfrentarse a las situaciones planteadas en las actividades?

\section{Marco teórico}

Muchas investigaciones se han realizado alrededor del uso de recursos tecnológicos en diferentes campos de las matemáticas escolares, como el álgebra (Guerrero, Rojano, Maviriks, \& Hoyles, 2011; Filloy, Puig, \& Rojano, 2008; Kieran, 2007; Guerrero, \& Rivera, 2002; Rojano, 2001), la geometría (Kuzniak \& Rauscher, 2011), o el cálculo (Aspinwall, Shaw \& Presmeg, 1997), estudiando los procesos y acercándose al aprendizaje con estas herramientas. Sin embargo, también es necesario estudiar lo que sucede en las interacciones entre los diferentes dominios específicos de las matemáticas (Kuzniak, 2012, 2013) con el fin de comprender el funcionamiento global del trabajo matemático. Kuzniak (2012) señala que este debe ser un ir y venir dentro de los diferentes espacios de trabajo matemático (ETM) que se presentan a los estudiantes en el aula. Dicha interacción puede darse en algunos ámbitos de manera más natural; 
es el caso, por ejemplo, de la geometría y la aritmética, donde a través del concepto de medida pueden conjugarse conceptos de ambos ETM.

Particularmente, en la investigación que se reporta analizamos la actividad realizada por los estudiantes de bachillerato en tareas que combinan el trabajo geométrico con el trabajo aritmético en Geogebra. Dicha combinación además permite pensar en un ETM como el contexto para promover el aprendizaje de conceptos en otro ETM; es decir, no resulta necesario "salir" del ámbito de las propias matemáticas para tratar de dar sentido y motivar el aprendizaje de las mismas. De igual forma, los medios tecnológicos y el software educativo que permiten mostrar, manipular y explorar diferentes representaciones de conceptos matemáticos (Duval, 1993), se han convertido en las herramientas idóneas para promover la integración de diferentes espacios de trabajo matemático (ETM).

Aunado al uso de la tecnología, en el aula es necesario crear verdaderos ambientes de trabajo matemático que promuevan el aprendizaje. Esta investigación retoma las ideas provenientes de múltiples investigaciones con relación a la generación de entornos de aprendizaje colaborativos y de trabajo individual. Particularmente, se habla de los Ambientes Tecnológico Interactivos para el Aprendizaje de las Matemáticas (ATIAM), término acuñado por Cortés y Núñez (2007) para describir los trabajos realizados en la línea de investigación sobre el uso de tecnología computacional para la enseñanza y el aprendizaje de las matemáticas, en la que también convergen aspectos y tendencias educativas actuales como: el desarrollo y uso de programas de cómputo, el uso de diversas plataformas de Internet y la utilización de calculadoras, entre otros, tanto desde la perspectiva de los profesores (didáctica y enseñanza) como desde el punto de vista del aprendizaje de las matemáticas (aspectos cognitivos) (Cortés, Guerrero, Morales \& Pedroza, 2014).

El uso de la tecnología en el aula de matemáticas modifica las relaciones entre los diversos actores que interactúan en ella; en ese sentido se ha definido un ATIAM como "aquel (ambiente) que se genera en el espacio o entorno donde los actores de los procesos de enseñanza y de aprendizaje (profesor y alumno) y el objeto de conocimiento, interactúan de forma organizada a través de una metodología que incluye actividades de aprendizaje con el uso de tecnología" (Cortés \& Núñez, 2007).

La investigación realizada alrededor de los ATIAM (Cortes \& Núñez 2007; Núñez \& Cortes 2008; Núñez 2008), ha mostrado que estos ambientes tecnológicos tienen potencial para 
favorecer, en los estudiantes, el desarrollo de habilidades para la construcción de procesos de aprendizaje y de conceptos matemáticos. Para la creación de un ATIAM es preciso contar con: 1) una propuesta teórica de enseñanza y/o aprendizaje; 2) actividades que faciliten y estimulen la construcción de aprendizajes; y, 3) una metodología de enseñanza acorde con los puntos anteriores (Núñez \& Cortés, 2008).

La investigación que se reporta tiene que ver con el aprendizaje de nociones de aproximación y estimación. Si bien, como se ha mencionado previamente, se hace uso de un espacio de trabajo geométrico (ETG) como contexto, el énfasis del análisis se da en términos de estas nociones.

Actualmente el currículo de matemáticas del nivel básico se centra en el desarrollo de competencias así como en el aprendizaje de conocimientos mediante la resolución de problemas. Dicho currículo ha relegado a segundo término algunas nociones básicas que resulta necesario retomar, ya que son parte de la alfabetización matemática requerida por todo ciudadano competente; es decir, forman parte del conocimiento fundamental (SEP, 2011). Una de ellas es la capacidad para hacer aproximaciones; en la vida diaria tenemos necesidad de hacerlo todos los días y de muchas formas: al manejar, estimamos velocidades; al ir al mercado, estimamos precios y medidas; nos darnos cuenta que nos equivocamos cuando hacemos una operación o juzgamos la validez de ciertas afirmaciones cuantitativas. Generalmente, hacemos estimaciones cualitativas y cuantitativas; sin embargo, muchos de los fenómenos que nos afectan se han vuelto tan complejos que no podemos percibirlos directamente o los tratamos de manera puramente cualitativa.

Si bien en el currículo actual se hacen diferentes recomendaciones para generar un alfabetismo funcional, del cual la aritmética es parte fundamental, la noción de aproximación es escasa en las situaciones que se presentan a los estudiantes, sobreponiendo a ella el uso de la calculadora de bolsillo como un auxiliar en la resolución de problemas.

Esta situación es compartida por los estudiantes en otros países; diferentes investigadores (Chang, et al. 2011; Gooya, et al., 2011; Hannighofer, et al., 2011; Smith, 2011) han resaltado la necesidad de incorporar al currículo actividades que favorezcan el desarrollo de estas habilidades. 


\section{Metodología}

Para el presente trabajo de investigación se diseñaron y adecuaron tres tareas a ser utilizadas como contexto de la actividad: Construyendo polígonos, Construcción de polígonos regulares utilizando triángulos isósceles y El problema de la caja. La primera de las mismas se aplicó a un grupo de 14 estudiantes de bachillerato y las dos restantes a un segundo grupo de alumnos. Hay que hacer notar que las actividades: Construyendo polígonos y Construcción de polígonos regulares a través de triángulos isósceles tratan el mismo tema, aunque con diferente enfoque y, por supuesto, con preguntas distintas, situación que nos da además la oportunidad de hacer una comparación entre las respuestas de los grupos.

La intervención educativa para la investigación se realizó con alumnos de la escuela preparatoria Melchor Ocampo, dependiente de la Universidad Michoacana de San Nicolás de Hidalgo y del Conalep II, Morelia.

El primer grupo de 14 estudiantes se conformó por jóvenes que de manera voluntaria decidieron participar en el estudio. Estos a su vez se dividieron (de manera voluntaria) en cinco grupos con 2 personas, un grupo de 1 persona y un grupo de 3 personas.

En el Conalep II, Morelia, se contó con diferente número de alumnos participantes en cada una de las actividades que realizamos: en las actividades de polígonos participaron 26 alumnos, quienes a su vez se repartieron en doce equipos de dos estudiantes y dos de un alumno. Mientras que para la actividad denominada El problema de la caja, participaron 13 alumnos que se dividieron en 6 equipos de 2 integrantes y en uno de 1.

Esta experimentación se realizó en el periodo del 3 de mayo al 17 de junio de 2013. El diseño y desarrollo de la misma se vinculó con una concepción de enseñanza y aprendizaje sustentado en el trabajo colaborativo, caracterizado por la aceptación compartida de responsabilidades mutuas de los miembros participantes en un equipo o en un grupo, en el que los roles del profesor y los alumnos son pieza fundamental. Asimismo, se relacionó el diseño y desarrollo del modelo interactivo con una reflexión individual acerca de los que se requería indagar.

A lo largo de la implementación se puso a disposición de los estudiantes y en todo momento:

1. Computadora de escritorio a la mano para manejo personal.

2. Software Geogebra instalado en la computadora de uso personal.

3. Un proyector, donde también se dio al alumno una introducción al manejo del Software Geogebra, además de explicarle algunas situaciones donde había duda general. 
4. Materiales convencionales (hojas de trabajo, hojas de cuadernos, pizarrón blanco y plumines).

Dado que los alumnos desconocían el software Geogebra, se les dio una pequeña introducción sobre su manejo, haciendo principal énfasis en las herramientas utilizadas en el desarrollo de la actividad.

Con los alumnos del Conalep II, Morelia, se trabajó durante tres sesiones de dos horas cada una, mientras que en la preparatoria Melchor Ocampo se les dio únicamente una sesión de dos horas.

\section{Exposición de la propuesta}

La propuesta consistió en la implementación de tres actividades en un ETG que, a través del concepto de medida, se combina con la noción aritmética de aproximación. Enseguida se explica con detalle una de ellas y, por cuestión de espacio, solamente se hacen comentarios a las otras. La actividad denominada Construcción de polígonos regulares usando triángulos isósceles, consta de tres secciones. La primera de ellas corresponde a un fundamento teórico donde se espera que el alumno, a través de contestar una serie de preguntas, sea capaz de recordar algunos conocimientos previos clave. La segunda parte de la actividad corresponde a tareas utilizando el software Geogebra en la que se proporciona una construcción geométrica que los estudiantes tienen que manipular. Se trata de la partición tradicional de un polígono regular en tantos triángulos isósceles congruentes como el número de lados del mismo. En esta se pide a los estudiantes trabajar con el ángulo central (el ángulo $\alpha$ de la figura 1) en casos de polígonos regulares especialmente complejos, como el de 7 lados, en el que dicho ángulo solo puede darse de manera aproximada debido a las limitaciones del sistema de cómputo; limitaciones que en este caso se aprovechan para generar una actividad significativa para los estudiantes, creando condiciones para realizar procesos de experimentación y permitiendo un trabajo de carácter exploratorio (Guerrero \& Cortés, 2013; Tanguay, Geeraerts, Saboya, Venant, Guerrero \& Morales, 2013).

Mediante ciertos cuestionamientos incluidos en una hoja de trabajo, se pide a los estudiantes que manipulen el ángulo y la medida del lado para, en un proceso de aproximación, logren cerrar lo mejor posible el polígono. 


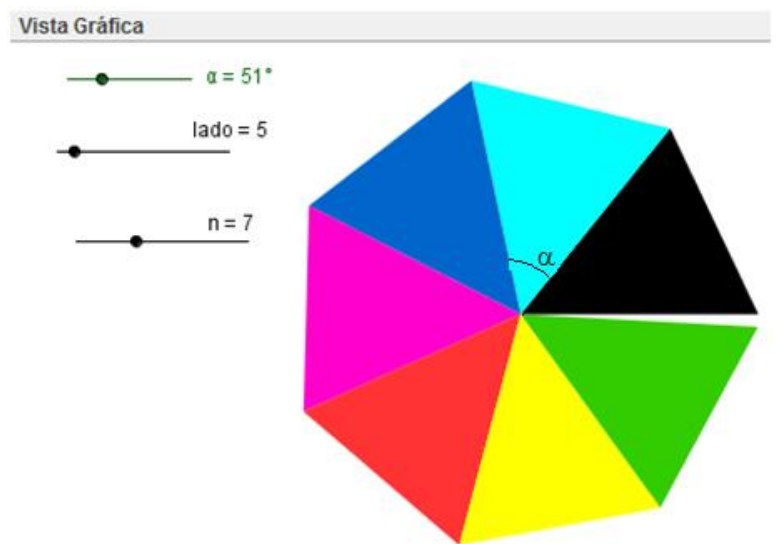

Figura 1. Construcción interactiva diseñada para la actividad de aproximación angular

De igual forma, era importante que el alumno obtuviera la relación de proporcionalidad inversa entre el número $\mathrm{n}$ de triángulos que se necesitan para construir un polígono y el ángulo $\alpha$ respectivo; esto es, $\quad n=\frac{k}{\alpha}$ con k constante y que reflexionara sobre la relación entre el ángulo $\alpha$ y la longitud l del lado.

La tercera sección de esta actividad les dio la oportunidad a los estudiantes de llegar a algunas conclusiones sobre los resultados obtenidos, por ejemplo, por qué no se puede construir un polígono regular de siete lados, o que investigaran sobre otros casos de polígonos regulares y sus propiedades métricas, como para los que es imposible realizar una construcción geométrica mediante la estrategia planteada.

La primera de las actividades aplicadas, denominada Construyendo Polígonos, trata también de la posibilidad o imposibilidad de construcción de polígonos regulares a partir de triángulos. En ella se proporciona a los estudiantes un archivo en el que pueden disponer de un conjunto hasta de 15 triángulos isósceles, todos congruentes con uno previamente determinado (ver figura 2). Con dichos triángulos se pide a los estudiantes construir polígonos uniendo los triángulos por vértices y lados. Los estudiantes pueden variar el ángulo en el vértice del triángulo isósceles, la medida de su base y el número de triángulos que requieren. En la hoja de trabajo correspondiente a esta actividad, se hacen preguntas acerca de cuánto deberá medir el ángulo en el vértice A del triángulo $\mathrm{ABC}$ para construir polígonos regulares con diferente número de lados, ya que la actividad trata de hacer al estudiante reflexionar sobre la posibilidad (o no) de construir polígonos regulares de la manera en que comúnmente se tratan dichas figuras en los libros de texto al hablar de su perímetro o de su área. 


\section{Exploración y análisis de poligonos}
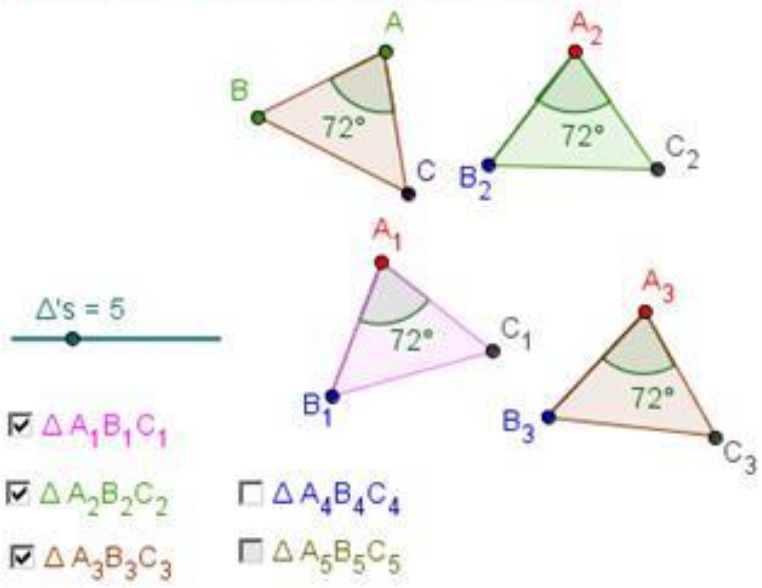

Figura 2. Actividad del archivo en Geogebra

Otra de las actividades denominada El problema de la caja, consiste en una simulación en Geogebra en la que los estudiantes tienen que manipular ciertas medidas como la altura de la caja de un camión de carga que transita por una calle con cierta pendiente (también manipulable), con el fin de que este pueda ser estacionado bajo un cobertizo. Se pretende que la experimentación (Guerrero \& Cortés, 2013) con el modelo virtual ayude a los estudiantes a mejorar sus habilidades de aproximación. Es claro que aunque la caja del camión tenga una medida un poco inferior a la altura del cobertizo, este no necesariamente puede entrar sin chocar en el techo. Los estudiantes deben manipular ya sea el ángulo de inclinación de la calle (el ángulo $\alpha$ de la figura 3) o la altura de la caja del camión con el fin de lograr la meta. Se pide, en una hoja de trabajo, hacer ciertas tareas relacionadas con la manipulación de las cantidades descritas para ajustar lo mejor posible las medidas.

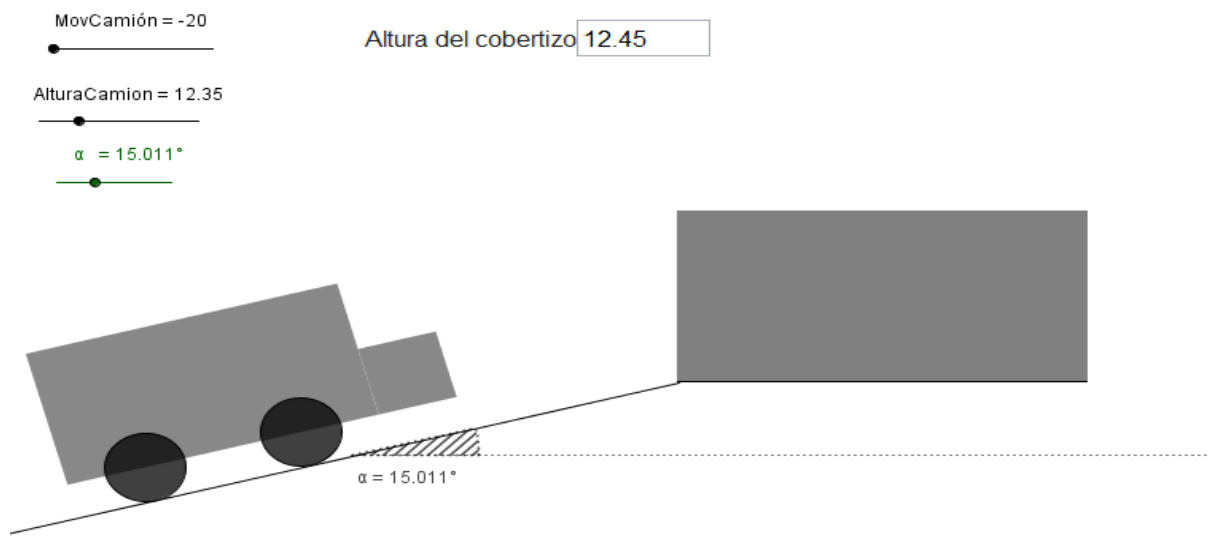

Figura 3. Construcción interactiva diseñada para la actividad de aproximación 


\section{Resultados}

Una de las características básicas de los alumnos que respondieron las actividades es el escaso nivel de profundidad del conocimiento geométrico, que se consideraba indispensable para trabajar en las actividades y el cual corresponde a un curso de geometría básica. Ejemplos considerados dentro de esta categoría son: los triángulos isósceles tienen dos lados iguales, la suma de los ángulos internos de cualquier triángulo es 180 grados, los ángulos en el triángulo equilátero son iguales, un polígono regular de $n$ lados tiene exactamente $n$ vértices. De igual manera, los alumnos informaron tener total desconocimiento en el uso de software de geometría dinámica, como Geogebra.

Otra circunstancia importante hallada al trabajar con los alumnos, fue que en la mayoría de las situaciones estos responden de forma muy sencilla (ingenua en ocasiones), englobando una sola idea. Esto es, los alumnos fueron incapaces de poner en marcha y combinar varias ideas para la solución de un mismo problema.

Por otro lado, los estudiantes pudieron responder correctamente a preguntas relativas a los ángulos en polígonos regulares en algunos casos; por ejemplo, en el caso del pentágono señalan que la medida del ángulo $\alpha$ es de $72^{\circ}$ "porque en total son $360^{\circ}$ y se dividen entre 5". Sin embargo, en casos como el del heptágono, los estudiantes muestran dificultades debido a que en dichos casos "no se tiene un valor exacto" al dividir el ángulo total $\left(360^{\circ}\right)$ por el número de lados (7). Una respuesta como la siguiente: "El polígono de 7 lados no es un polígono regular porque le falta cerrarse", muestra que los estudiantes manejan los conceptos solamente a nivel perceptivo.

En la tabla 1 se muestran algunos resultados obtenidos del trabajo de los estudiantes con relación a la construcción presentada en la primera actividad aplicada, en donde con base en la manipulación de un conjunto de triángulos isósceles congruentes, se pide a los estudiantes la construcción de un polígono regular. Una respuesta típica se muestra en la figura 4.

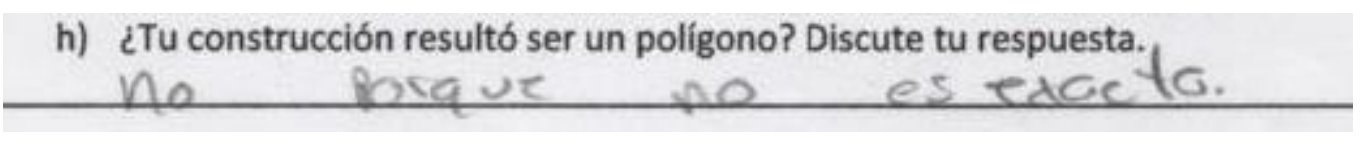

Figura 4. Respuesta típica de los estudiantes

Como se puede observar, los alumnos logran establecer por sí mismos el siguiente resultado importante en términos de construcción: la imposibilidad de construir geométricamente ciertos 
polígonos. Asimismo, se pudo observar la confusión que tienen con respecto a algunos conceptos matemáticos, por ejemplo, el de proporcionalidad.

Tabla I. Parte de las respuestas de los equipos a la actividad Construyendo polígonos.

\begin{tabular}{|l|l|}
\hline Equipo 1, sec. 424 & No, porque le faltó cerrarse. \\
\hline Equipo 2, sec. 424 & No, porque sobran partes en el polígono. \\
\hline Equipo 3, sec. 424 & No, tenía siete lados aunque uno era de menor tamaño. \\
\hline Equipo 4, sec. 424 & No, porque no es exacto. \\
\hline Equipo 1, sec. 403 & Si, pues cuando vamos cambiando las medidas, cambias los ángulos \\
& y salen diferentes figuras, polígonos regulares e irregulares. \\
\hline Equipo 2, sec. 403 & No, porque tiene 7 lados iguales y no es proporcional. \\
\hline Equipo 3, sec. 403 & Sí, por los lados que obtuvimos. \\
\hline Equipo 4 y 5, sec. 403 & Sí. \\
\hline Equipo 6 y 7, sec. 403 & No respondieron. \\
\hline
\end{tabular}

En el caso del heptágono, los estudiantes logran hacer aproximaciones para el ángulo solamente hasta la tercera cifra decimal del ángulo $\alpha$ sin que puedan cerrar completamente el polígono. No logran dar explicaciones pertinentes a este fenómeno ni ir más allá en las aproximaciones. Sus respuestas a preguntas relativas a la exactitud de resultados son muy básicas, englobando solamente una idea geométrica perceptiva sin conexión con los valores numéricos que obtienen.

\section{Conclusiones}

En el currículo de matemáticas se establece que existe un polígono regular de n lados para cada entero $\mathrm{n}>2 \mathrm{y}$, para cada uno de ellos, el ángulo central mide $\frac{360}{n}$ grados. Se espera también que el estudiante comprenda que ciertas fracciones no pueden expresarse de manera exacta en una forma decimal finita, y que no siempre es posible utilizar el signo de igualdad entre una fracción de la forma $\frac{m}{n}$ y una escritura decimal finita que represente lo mismo. Se insiste en la necesidad de utilizar diferentes formas de escritura de los números y que dichas formas serán útiles en el 
trabajo matemático posterior; sin embargo, estas ideas se dan de manera aislada, de tal forma que generan dificultades conceptuales en el aprendizaje de los estudiantes como lo muestra esta investigación.

Si bien los estudiantes que participaron en la presente investigación estuvieron interesados en realizar las actividades y trabajaron en ellas a lo largo de todas las sesiones sin tener dificultades en el manejo del software (aun cuando señalaron que nunca habían trabajado con un SGD), mostraron dificultades para seguir un argumento lógico y sus ideas fueron muy intuitivas y carentes de formalismo. Los estudiantes no trataron de comprobar sus respuestas por otros medios y además se observaron respuestas contradictorias en diferentes situaciones. Estas dificultades muestran que los estudiantes tienen problemas aun con nociones muy básicas, como las asociadas a los polígonos regulares.

Por otro lado, el software Geogebra posibilitó estudiar la abertura que queda en algunos polígonos "regulares"; haciendo que los estudiantes se percaten de este tipo de dificultades de construcción. Fue un importante auxiliar que ayudó a obtener información de manera experimental. 


\section{Bibliografía}

Aspinwall, L., Shaw, K. \& Presmeg, N. (1997). Uncontrollable Mental Imagery: Graphical Connections between a Function and its Derivative. Educational Studies in Mathematics, 33(3), pp. 301-317.

Cortés, C., Guerrero, L., Morales Ch., \& Pedroza, L. (2014) Tecnologías de la Información y la Comunicación (TIC): Aplicaciones Tecnológicas para el Aprendizaje de las Matemáticas. En: (2014) Agustín Carrillo (Ed.) Unión. Revista Iberoamericana de Educación Matemática. No 39. Septiembre 2014, pp. 141-161.

Cortés, C. \& Núñez, E. (2007) Ambientes tecnológicos interactivos para el aprendizaje de las matemáticas. Memorias del IX Congreso Nacional de Investigación Educativa. México, 2007.

Chang, K., Males, L., Mosier, A. \& Ginulates, F. (2011) Exploring US textbooks' treatment of the estimation of linear measurements. ZDM, 43(5), pp. 697-708.

Duval, R. (1993) Registres de représentation sémiotique et fonctionnement cognitif de la pensée. Annales de Didactique et de Sciences Cognitives, n5, p. 37-65. IREM de Strasbourg.

Filloy, E., Puig, L. \& Rojano. T. (2008). Educational algebra. A theoretical and empirical approach. Springer, USA.

Gooya, S., Khosroshahi, L. \& Teppo, A. (2011) Iranian students' measurement estimation performance involving linear and area attributes of real-world objects. ZDM, 43(5), pp. 709-722.

Guerrero, L. \& Cortés, C. (2013) Ambientes tecnológico-interactivos para el aprendizaje de las matemáticas: investigaciones y experiencias en geometría y cálculo. En: Rojano, T. (Editora) Las tecnologías digitales en la enseñanza de las matemáticas. Ed. Trillas, México.

Guerrero, L. \& Rivera, A. (2002) Exploration of patterns and recursive functions. En: Proceedings of the 24th PME-NA Conference, vol. 1. Athens, GA, USA.

Guerrero, L., Rojano, T., Maviriks, M. \& Hoyles, C. (2011). Critical Moments in generalization tasks. Building algebraic rules in a digital sign system. En: Wiest, L. \& Lamberg, T. (Eds.) Proceedings of the 33rd Annual Meeting of the North American Chapter of the 
International Group for the Psychology of Mathematics Education. Reno, NV: University of Nevada, Reno.

Hannighofer, J., Van den Heuvel-Panhuizen, M., Weirich, S. \& Robitzsch, A. (2011) Revealing German primary school students' achievement in measurement. ZDM, 43(5), pp. 651665.

Kieran, C. (2007). Learning and teaching algebra at the middle school through college levels: Building meaning for symbols and their manipulation. En: Lester, Jr., (Ed.), Second handbook of research on mathematics teaching and learning (pp. 707-762). Greenwich, CT: Information Age Publishing.

Kuzniak, A. (2012) Understanding the nature of the geometric work through its developement and its transformations. 12th International Congress on Mathematical Education, COEX, Seoul, Korea.

Kuzniak, A. (2013) Travail mathématique et domaines mathématiques. To appear in A. Kuzniak et P. R. Richard (eds), Proceedings of the 3rd symposium Espace de Travail Mathématique. Université de Montréal.

Kuzniak, A. \& Rauscher, J. (2011) How do teachers' approaches to geometric work relate to geometry students' learning difficulties? Educational Studies in Mathematics, Vol. 77, pp.129-147.

Núñez, E. \& Cortés, C. (2008) Propuesta de una metodología de enseñanza usando ambientes tecnológicos interactivos. En: R. Pantoja, E. Añorve, C. Cortés y L. Osornio (Editores) Investigaciones y propuestas sobre el uso de la tecnología en educación matemática. Vol. 1, 121-231, Editorial AMIUTEM.

Rojano, T. (2001). Algebraic Reasoning with Spreadsheets. Proceedings of the International Seminar "Reasoning explanation and proof in school mathematics and their place in the intended curriculum". Qualificactions and Curriculum Authority. Cambridge, UK, pp. 116.

SEP (2011). Programas de estudio 2011. Guía para el Maestro. Educación Básica. Secundaria. Matemáticas. Subsecretaría de Educación Básica de la SEP, México.

Smith, J., Van den Heuvel-Panhuizen, M. \& Teppo, A. (2011) Learning, teaching, and using measurement: introduction to the issue. ZDM, Zentralblatt für Didaktik der Mathematik, 43, pp. 617-620. 
Tanguay, D., Geeraerts, L., Saboya, M., Venant, F., Guerrero, ML. \& Morales, Ch. (2013) An activity entailing exactness and approximation of angle measurement in a DGS. CERME 8. 6-10 de febrero, Turquía. 